\title{
Sistema Embebido de Análisis y Control por Medio de Visión Artificial y Mediciones Ultrasónicas, Basado en LabVIEW y Hardware roboRIO
}

\section{Embedded System of Analysis and Control Through Artificial Vision and Ultrasonic Measurements, Based on LabVIEW and roboRIO Hardware}

PONCE-MELLADO, Juan Jorge †*, SILVA-JUÁREZ, Alejandro y SALAZAR-PEDRAZA, Miguel de Jesús

Universidad Tecnológica De Puebla

ID $1^{\text {er }}$ Autor: Juan Jorge, Ponce-Mellado / ORC ID: 0000-0003-2186-2868, Researcher ID Thomson: G-6040-2018, arXiv Author ID: Jorge_Ponce, CVU CONACYT ID: 900658

ID $1^{\text {er }}$ Coautor: Alejandro, Silva-Juárez / ORC ID: 0000-0001-8473-9803, Researcher ID Thomson: F-6969-2018, arXiv Author ID: Alejandro_Silva, CVU CONACYT ID: 637028

ID $2^{\text {do }}$ Coautor: Miguel de Jesús, Salazar-Pedraza / ORC ID: 0000-0001-9210-1930, Researcher ID Thomson: Q-98582018, arXiv Author ID: EduCIBEL, CVU CONACYT ID: 615713

\section{Resumen}

En mantenimiento es vital conocer el estado físico de un equipo o proceso en tiempo real de manera eficaz, rápida y confiable, ya que, se evitaría las fallas a corto, mediano o largo plazo, provocadas por la mala operación o desgaste de piezas que conforman el proceso o equipo. Una de las herramientas en que permite esto se le conoce como; "Mantenimiento Predictivo", en donde, es indispensable el uso de nuevas tecnologías de procesamiento y análisis. La implementación de un sistema de monitoreo por visión artificial en mantenimiento eliminara la necesidad de diagnosticar de manera presencial partes de un equipo o proceso. Por ello, se diseñó e implemento un sistema embebido de visión artificial y mediciones ultrasónicas, utilizando la plataforma de LabVIEW y hardware RoboRIO, capaz de; analizar, monitorear, clasificar, contabilizar y procesar el espectro electromagnético visible de un número $\mathrm{N}$ de objetos con dimensiones $(\mathrm{X}, \mathrm{Y}$, $\mathrm{Z}$ ) en un proceso de producción. El cual logra una efectividad del $87 \%$ contra un sistema ideal, en el que no existe error alguno. Siendo está, la primera de 3 fases de un sistema más complejo de monitoreo de visión artificial para el diagnóstico de equipos y procesos en mantenimiento.

Visión artificial, Monitoreo, Sistema embebido

\begin{abstract}
In maintenance is vital to know the physical state of a equipment or process in real time effectively, fast and reliable way, since it would avoid short, medium or long term failures, caused by the bad operation or wear of parts that make up the process or equipment. One of the tools in which he allows this is known as; "Predictive maintenance", where the use of new processing and analysis technologies is indispensable. The implementation of a monitoring system by artificial vision in maintenance will eliminate the need to diagnose in person parts of a team or process. For this reason, an embedded system of artificial vision and ultrasonic measurements was designed and implemented, using the LabVIEW platform and RoboRIO hardware, capable to; analyze, monitor, classify, record and process the visible electromagnetic spectrum of a number $\mathrm{N}$ of objects with dimensions $(\mathrm{X}, \mathrm{Y}, \mathrm{Z})$ in a production process. Which achieves an effectiveness of $87 \%$ against an ideal system, in which there is no error. Being this is the first of 3 phases of a more complex artificial vision monitoring system for the diagnosis of maintenance equipment and processes.
\end{abstract}

Artificial vision, Monitoring, Embedded system

Citación: PONCE-MELLADO, Juan Jorge, SILVA-JUÁREZ, Alejandro y SALAZAR-PEDRAZA, Miguel de Jesús. Sistema Embebido de Análisis y Control por medio de Visión Artificial y Mediciones Ultrasónicas, basado en LabVIEW y Hardware roboRIO. Revista de Tecnologías en Procesos Industriales. 2019 3-8: 21-28

\footnotetext{
* Correspondencia del Autor (juan.ponce@utpuebla.edu.mx)

$\dagger$ Investigador contribuyendo como primer autor.
} 


\section{Introducción}

De acuerdo con una investigación publicada por el MIT technology review en 2008; un $47 \%$ de los procesos que se efectuaban manualmente, han sido automatizados durante los últimos 10 años, a consecuencia de requerir procesos más eficientes, con mayor precisión, más rápidos y seguros.

Debido a esto, actualmente se requiere sistemas autómatas que permitan mayor confiabilidad, en los que se deje atrás la automatización fija, y que se monitoree constantemente el proceso en tiempo real, como ejemplo: sistemas automatizados remotos, sistemas basados en redes neuronales, sistemas SCADA o sistemas embebidos de visión artificial y sensores de alta precisión.

Este último se emplea muy a menudo para la detección de: patrones, códigos de barras, formas, dimensiones, colores, posiciones, entre otras aplicaciones.

Para nuestro caso en particular el diseño de este sistema embebido de visión artificial, cubre satisfactoriamente las áreas de aplicación ya mencionadas, con la excepción de identificación de patrones y formas.

De lo anterior se puede destacar el hecho que la automatización no solo puede ser empleada para el control autómata de procesos, si no, también en la tarea constante de mantenimiento, al tener sistemas autómatas, se podrá abarcar con mayor facilidad y eficiencia los objetivos diarios a los que se enfrenta mantenimiento, como la detección de fallas tempranas, comportamiento anormal de máquinas, etc.

Por ello, se diseñó e implemento un sistema embebido de visión artificial y mediciones ultrasónicas, utilizando la plataforma de LabVIEW y hardware RoboRIO, capaz de; analizar, monitorear, clasificar, contabilizar y procesar el espectro electromagnético visible de un número $\mathrm{N}$ de objetos con dimensiones $(\mathrm{X}, \mathrm{Y}, \mathrm{Z})$ en un proceso de producción, para así lograr un $87 \%$ de efectividad en él análisis, contra un sistema ideal, en el que no existe error alguno.
Siendo está, la primera de 3 fases de un sistema más complejo de monitoreo de visión artificial para el diagnóstico de equipos y procesos en mantenimiento, ya que en esta primera fase, solo se enfocara al análisis de dimensiones y colores de objetos, posterior mente en la segunda fase, se pretende alcanzar el reconocimiento de patrones, por medio de redes neuronales, para así alcanzar la tercera y última fase, donde se implementara el sistema embebido en el reconocimiento patrones en fallas de rodamientos, engranes y bandas, además de poder analizar el comportamiento de una maquina y saber si es el óptimo o no.

Para entender con más claridad cuál es el alcance y las partes que conforma este sistema de embebido, se dividirá el estudio en tres partes principales, las cuales son:

1. Banco de pruebas; donde se analizará las partes físicas que se emplearon para el desarrollo de este sistema embebido; hardware, estructuras, sensores, actuadores, etc.

2. Adquisición y procesamiento de señales de visión artificial (imagen) y ultrasónicas; en este apartado se explicará a detalle los algoritmos, ecuaciones y programas desarrollados para el análisis y procesamiento de imágenes y señales ultrasónicas.

3. Diseño e implementación del algoritmo de control para el sistema embebido; se hablará del programa principal de control, la interfaz de usuario y los algoritmos de combinatoria para el procesamiento de diferentes variables en los objetos, además se explicará el patrón de programación empleado para que programa general utilizara los datos adquiridos por el programa analizado en la sección 2.

Las ventajas más claras con las que cuenta este sistema embebido es el hardware roboRIO, ya que, cuenta con un procesador FPGA, dando así la posibilidad de adquirir y procesar un gran número de datos adquiridos.

Los arreglos de compuertas programables en campo (FPGAs) son chips de silicio reprogramables. Ross Freeman, el cofundador de Xilinx, inventó el primer FPGA en 1985. 
La adopción del chip FPGA en las industrias es guiada por el hecho que los FPGAs combinan lo mejor de los circuitos integrados de aplicación específica (ASICs) y sistemas basados en procesador. Los FPGAs ofrecen velocidades temporizadas por hardware $\mathrm{y}$ fiabilidad, pero sin requerir altos volúmenes de recursos para compensar el gran gasto que genera un diseño personalizado de ASIC, (National Instruments, 2012).

\section{Objetivo}

Diseñar e implementar un sistema embebido de visión artificial y mediciones ultrasónicas, utilizando la plataforma de LabVIEW y hardware RoboRIO, capaz de; analizar, monitorear, clasificar, contabilizar y procesar el espectro electromagnético visible de un número $\mathrm{N}$ de objetos con dimensiones $(\mathrm{X}, \mathrm{Y}, \mathrm{Z})$ en un proceso de producción, para así lograr un $87 \%$ de efectividad en él análisis.

\section{Metodología}

La metodología que se empleó en el desarrollo e implementación de este sistema embebido se pueden observar en el Figura 1.

\begin{tabular}{|c|c|}
\hline \multicolumn{2}{|c|}{ INICIO } \\
\hline \multirow[t]{2}{*}{$\begin{array}{c}\text { Análisis del } \\
\text { proceso }\end{array}$} & $\begin{array}{l}\text { Se analizo a detalle las } \\
\text { características con las que } \\
\text { debería contar el sistema } \\
\text { embebido en función a las } \\
\text { necesidades del proceso. }\end{array}$ \\
\hline & $\begin{array}{l}\text { Estado del arte acerca de los } \\
\text { métodos y tecnologías en } \\
\text { sistemas embebidos. }\end{array}$ \\
\hline Factores de diseño & $\begin{array}{l}\text { Factores y características a } \\
\text { considerar para el diseño de un } \\
\text { sistema embebido. }\end{array}$ \\
\hline $\begin{array}{c}\text { Diseño e } \\
\text { Implementación }\end{array}$ & $\begin{array}{l}\text { Las partes que conforma este } \\
\text { sistema de embebido se } \\
\text { dividirán en tres partes } \\
\text { principales, las cuales son: banco } \\
\text { de pruebas, adquisición y } \\
\text { procesamiento de señales de } \\
\text { visión artificial (imagen) y } \\
\text { ultrasónicas, y, por último, } \\
\text { diseño e implementación del } \\
\text { algoritmo de control para el } \\
\text { sistema embebido. }\end{array}$ \\
\hline $\begin{array}{l}\text { Pruebas y } \\
\text { Resultados }\end{array}$ & $\begin{array}{l}\text { Se realizaron las pruebas } \\
\text { correspondientes del sistema } \\
\text { embebido y se comparó los } \\
\text { resultados esperados contra los } \\
\text { obtenidos. }\end{array}$ \\
\hline & FIN \\
\hline
\end{tabular}

Figura 1 Metodología empleada

\section{Desarrollo}

\section{Banco de Pruebas}

El diseño del banco de pruebas debe de cumplir las siguientes características las cuales satisfacen los requerimientos en el objetivo del proyecto:

- $\quad$ Hardware de control RoboRIO.

- $\quad$ Sistema de visión artificial y medidas ultrasónicas.

- Objetos o productos con medidas $(\boldsymbol{X}, \boldsymbol{Y}, \boldsymbol{Z})$ variables y diferentes colores.

- $\quad$ Sistema de transporte de productos por medio de una cinta transportadora.

Actuador clasificador de productos.

\section{Hardware de control RoboRIO}

El controlador roboRIO, cuenta con procesador FPGA de Xilinx Zynq-7020 como característica principal, lo que permite analizar y procesar en tiempo real los datos adquiridos. Además, las múltiples entradas y salidas digitales, permiten conectar un múltiple número de sensores y actuadores, los cuales funcionen en conjunto y en paralelo con el procesamiento de imágenes adquiridas por la cámara.

\section{Sistema de visión artificial $y$ medidas ultrasónicas}

Para el sistema de visión artificial es un conjunto de dos partes principales, primera; el sistema de procesamiento de la imagen (roboRIO), segunda; sistema de captura la imagen, el cual cuenta con una cámara WEB hp resolución 240 x 320 pixeles.

Para las medidas ultrasónicas se empleó un sensor ultrasónico HC-SR04, el cual nos permite tener medidas de $\pm 3 \mathrm{~mm}$ de error en las medidas que estemos realizando.

\section{Objetos o productos con medidas $(X, Y, Z)$ variables y diferentes colores}

Los objetos empleados cubren diferentes características en dimensiones y colores. Para dimensiones 4 alturas, 4 largos y una anchura, mientras que, en color se tienen 4 diferentes colores. Dando así un total de 64 posibles combinaciones de características en los distintos objetos, Ver Tabla 1. 


\begin{tabular}{|c|c|}
\hline Altura $(Z)$ & $\operatorname{Largo}(Y)$ \\
\hline $61,36,23$ y $17 \mathrm{~mm}$ & $114,88.6,63$ y $38 \mathrm{~mm}$ \\
\hline $\operatorname{Anchura}(X)$ & Color \\
\hline $20 \mathrm{~mm}$ & \\
\hline
\end{tabular}

Tabla 1 Características físicas y de dimensión de objetos

\section{Sistema de transporte de productos por} medio de una cinta transportadora

El sistema de transporte de los objetos tiene dimensiones de $80 \mathrm{~cm}$ de largo, $20 \mathrm{~cm}$ de anchura y $35 \mathrm{~cm}$ de alto, ver Figura 2. Para la estructura se empleó equipo de construcción VEX Robotics, y para el movimiento de la banda transportadora se empleó, un motor DC con moto reductor con alimentación de $7.2 \mathrm{~V}$, generando así un torque de $1.67 \mathrm{~N} \cdot \mathrm{m}$ a 100 RPM.

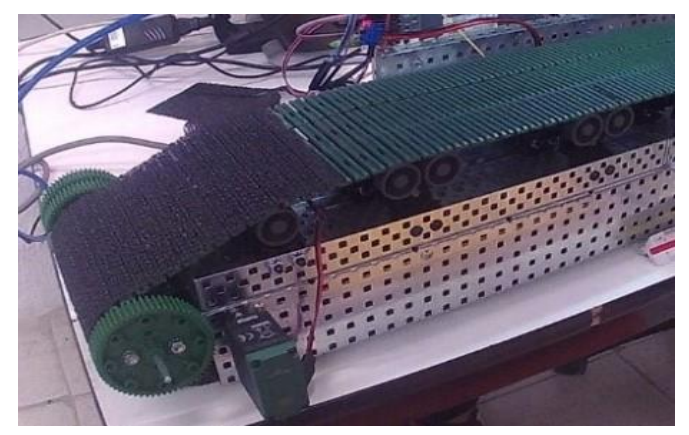

Figura 2. Estructura del sistema de transporte, banda transportadora

\section{Actuador clasificador de productos}

Para la clasificación y discriminación de los objetos se empleó un sistema en conjunto conformado por dos partes, primera; un motor DC con moto reductor con alimentación de $7.2 \mathrm{~V}$, generando así un torque de $1.67 \mathrm{~N} \cdot \mathrm{m}$ a 100 RPM, segunda; Un sistema mecánico de movimiento, el cual convierte el movimiento rotativo del motor DC en un movimiento rectilíneo, gracias a un sistema de engranecremallera, ver Figura 3.

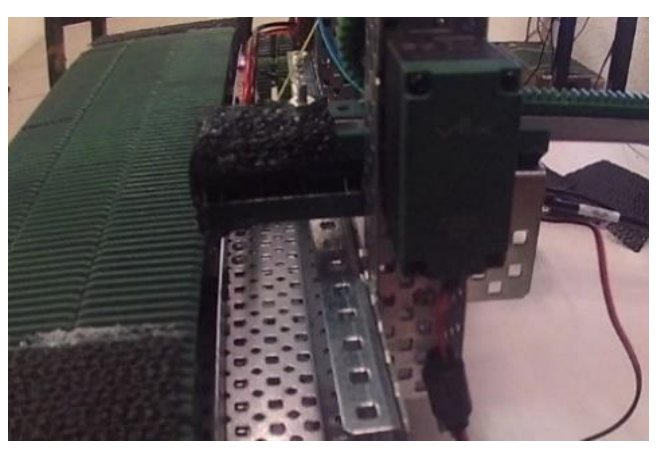

Figura 3 Sistema de engranaje cremallera

\section{Adquisición y procesamiento de señales de} visión artificial (imagen) y ultrasónicas

En la Figura 4 se muestra un algoritmo general de adquisición de señales en LabVIEW, ya sea para adquirir o generar señales.

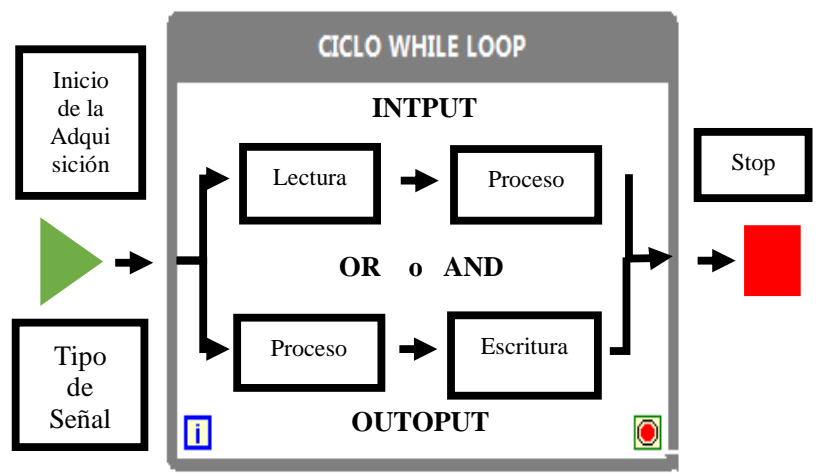

Figura 4 Algoritmo general de adquisición de señales en LabVIEW

La adquisición de las señales de visión y ultrasónicas, se emplea el algoritmo general que se muestra en la Figura 4, el cual se modifica para el caso particular en cada una de las adquisiciones de señales.

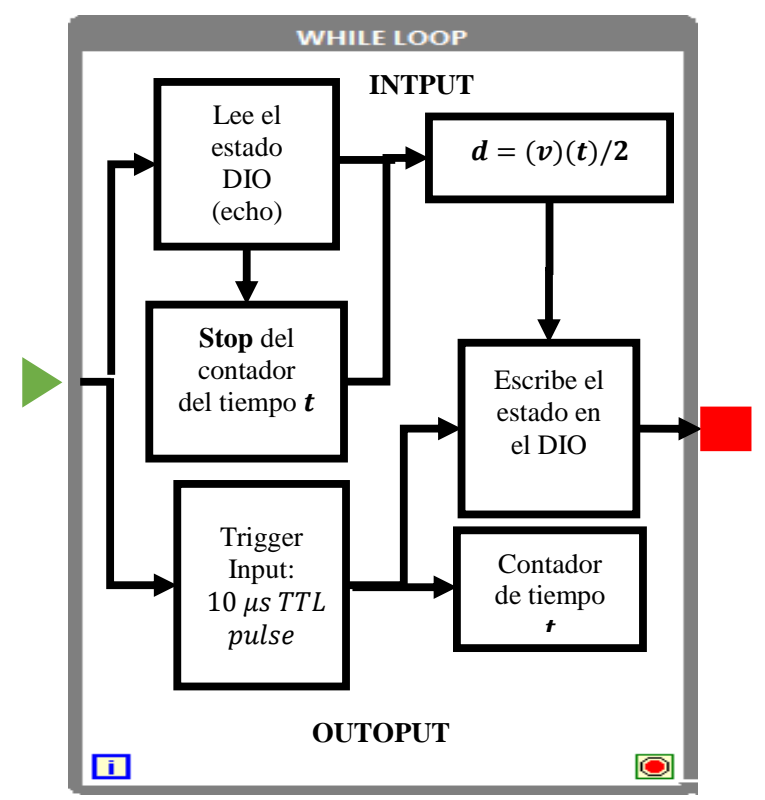

Figura 5 Algoritmo particular de adquisición de señales ultrasónica

En el caso particular del procesamiento de señales ultrasónicas reescribimos el algoritmo general a particular de la siguiente manera, ver Figura 5, este algoritmo tiene como propósito medir dimensiones de altura en el eje $(\boldsymbol{Z})$ de cualquier objeto o producto que sea procesado.

Para el caso particular del algoritmo de procesamiento de visión artificial por medio de una cámara y hardware roboRIO, se emplea el siguiente algoritmo desarrollado, ver Figura 6.

PONCE-MELLADO, Juan Jorge, SILVA-JUÁREZ, Alejandro y SALAZAR-PEDRAZA, Miguel de Jesús. Sistema Embebido de Análisis y Control por medio de Visión Artificial y Mediciones Ultrasónicas, basado en LabVIEW y Hardware roboRIO. Revista de Tecnologías en Procesos Industriales. 2019 


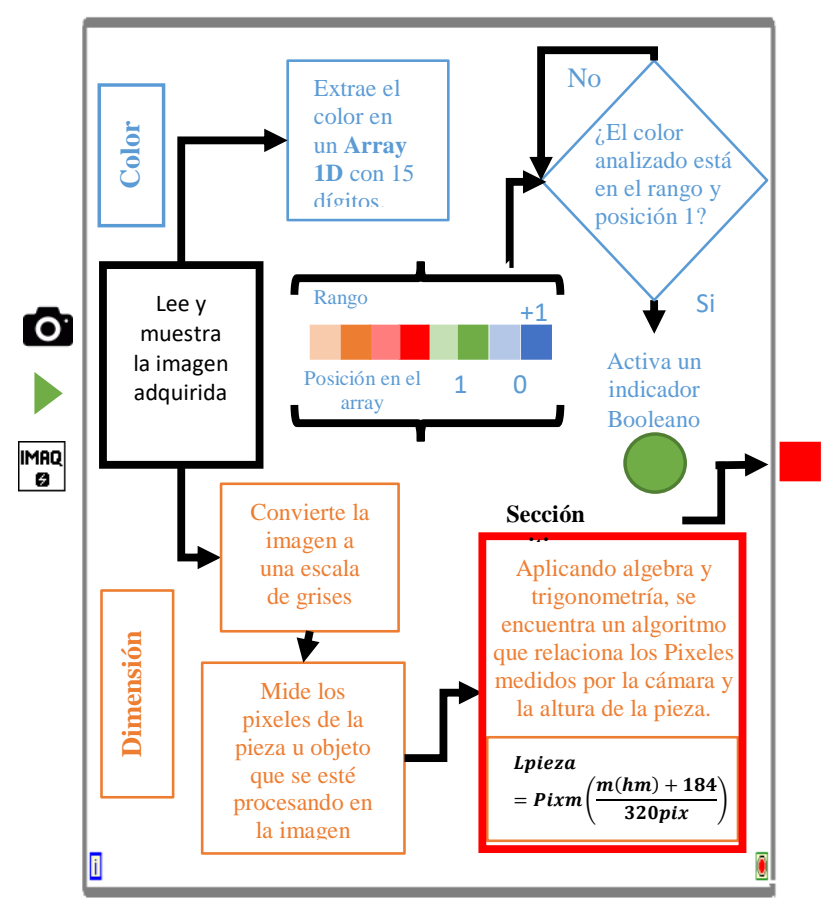

Figura 6 Algoritmo particular para el procesamiento de imágenes por visión artificial

El algoritmo de visión artificial se divide en dos partes principales; la primera sección tiene la función de analizar y procesar el espectro electromagnético visible. Para la segunda parte del algoritmo tiene como propósito el análisis dimensional de cada objeto, en específico las dimensiones $(\boldsymbol{X}, \boldsymbol{Y})$, (Largo, ancho). Para analizarlo utilizaremos el grafico que se muestra en la Figura 7, el cual es una analogía del campo de visión de cualquier cámara. Utilizando el concepto matemático de la recta y de trigonometría, relacionamos la longitud $L_{\text {pieza }}$ con respecto a la altura del objeto $h_{m}$ y los pixeles que mide la cámara $P i x_{m}$, esta relación se escribe de la siguiente manera, ver Ec. 1.

$$
L_{\text {pieza }}=\operatorname{Pix}_{m}\left(\frac{\left(-\frac{184 \mathrm{~mm}}{240 \mathrm{~mm}}\right)(\mathrm{hm})+184}{320 \text { pix }}\right)
$$

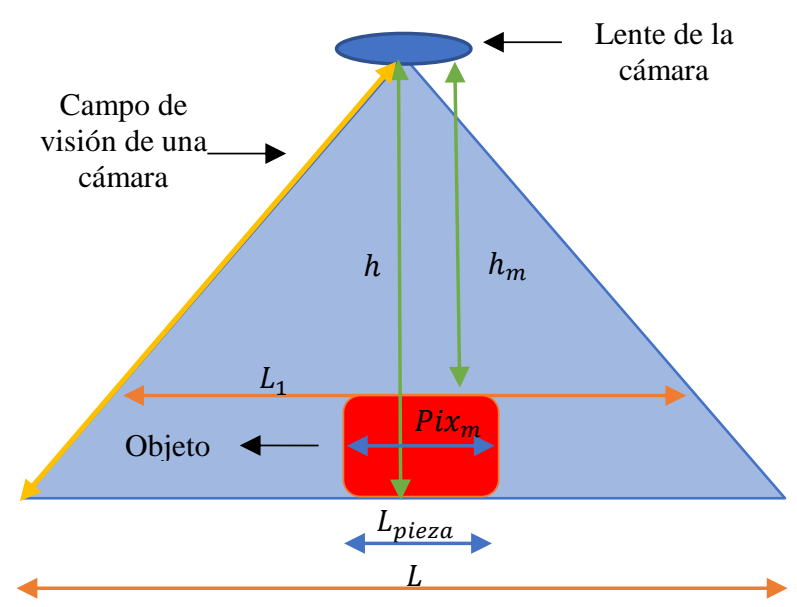

Figura 7 Relación de medidas de un objeto, con respecto al campo de visión de una cámara

\section{Donde:}

$$
\begin{aligned}
& L_{\text {pieza }} \text { Es la longitud real de la pieza u } \\
& \text { objeto en } \mathrm{mm} \text {. } \\
& \text { Son los pixeles medidos por la } \\
& \mathrm{Pix}_{m} \quad \text { cámara, los cuales tiene un rango de } \\
& 0 \text { a } 320 \text { pixeles. }
\end{aligned}
$$

Recapitulando, se desarrolló en LabVIEW el algoritmo de mediciones ultrasónicas y de visión artificial en un solo. El cual, aplicando la sintaxis propia de LabVIEW, se reescribe estos dos algoritmos en el siguiente; ver Figura 8.

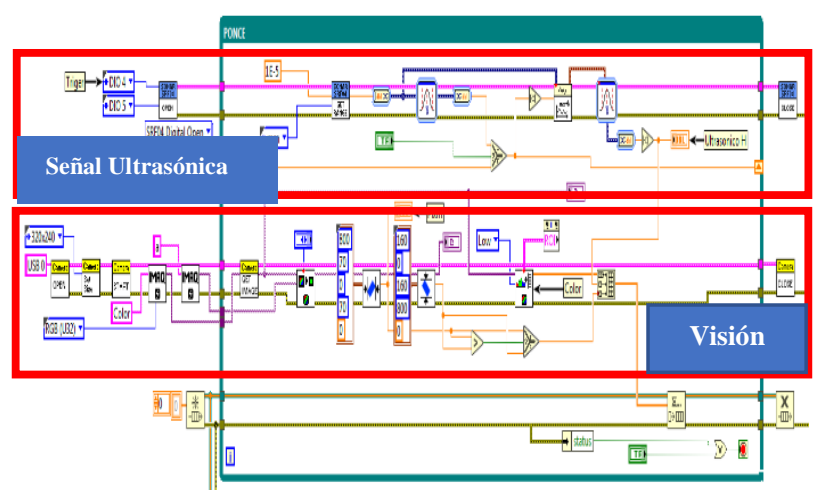

Figura 8 Sección del programa en LabVIEW para el procesamiento de imágenes por visión artificial

\section{Diseño e implementación del algoritmo de control para el sistema embebido}

En la Figura 9 se muestra la interfaz de usuario del sistema embebido, esta interfaz se divide en 6 partes principales, las cuales detallaremos a continuación, ver Tabla 2. 


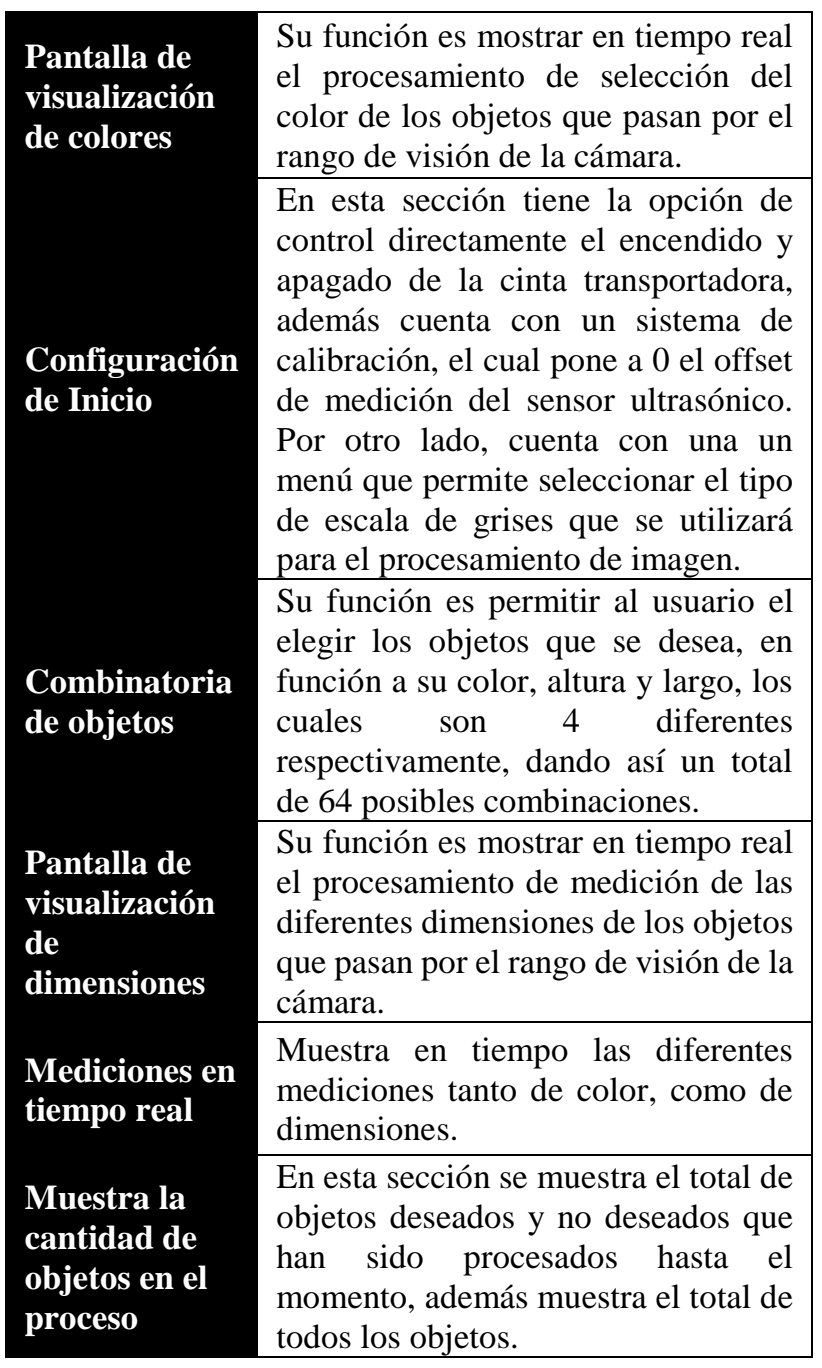

Tabla 2 Características físicas y de dimensión de los objetos

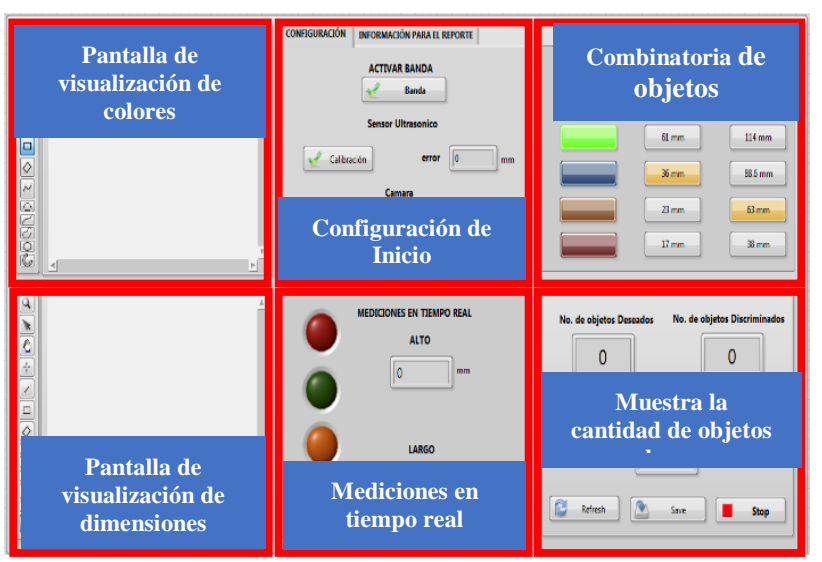

Figura 9 Secciones de la interfaz para el usuario del sistema embebido desarrollada en LabVIEW

El programa principal es una combinación de tres secciones; la primera es la adquisición de las señales ultrasónicas y de imagen por visión artificial, Segunda sección; es el algoritmo principal cuya función es el realizar la combinatoria entre las diferentes medidas y colores, la tercera sección; es la emisión de señales digitales elegidas por el programa principal a los diferentes actuadores, ver Figura 10 y 11.

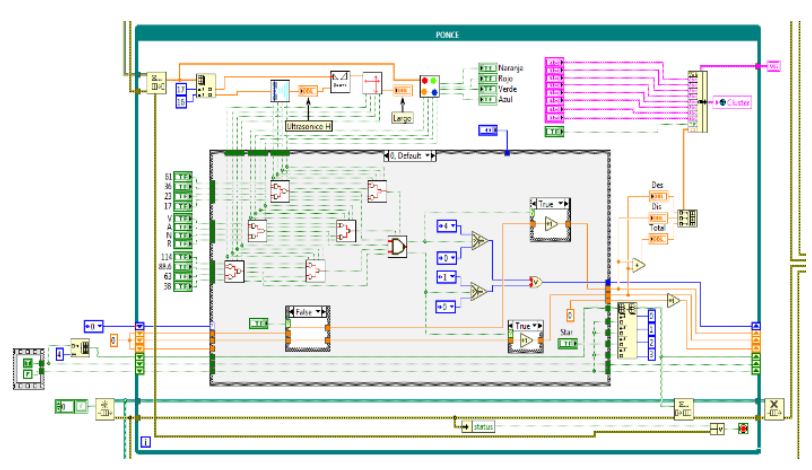

Figura 10 Sección principal del programa en LabVIEW; procesamiento y control del sistema embebido

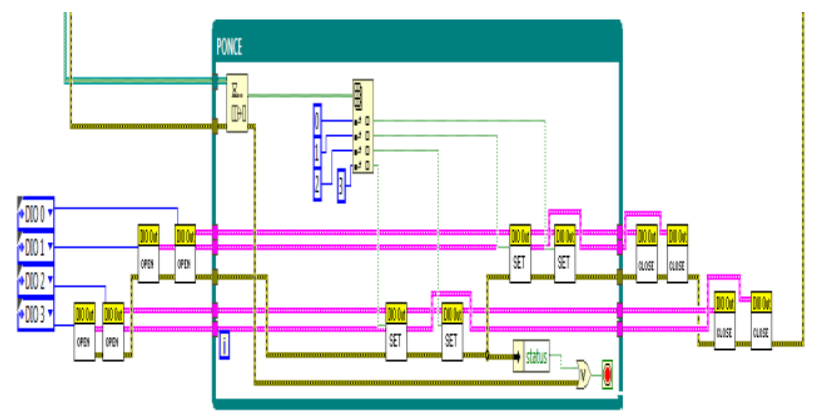

Figura 11 Sección del programa en LabVIEW para control de los actuadores

Para especificar como es la forma de comunicación entre los tres programas o algoritmos mencionados, se utilizó una estructura propia de LabVIEW, la cual se emplea para la comunicación entre bucles paralelos, cuya denotación es; Patrón Productorconsumidor.

\section{Resultados}

El Gráfico 1 se compara el tiempo que tarda en analizar el sistema embebido con 20 objetos, con respecto sistema ideal que, de igual manera analizara a 20 objetos, pero sin considerar el tiempo que se pierde al analizar un objeto incorrectamente.

Donde; $\boldsymbol{m}_{\boldsymbol{I}}$, es el ritmo de cambio que hay entre el número de objetos analizados para el caso ideal, con respecto al tiempo, y $\boldsymbol{m}_{\boldsymbol{r}}$, es el ritmo de cambio entre el número de objetos analizados para el caso real, con respecto al tiempo. $\Delta \boldsymbol{t}$ es la diferencia de tiempo de análisis entre el caso ideal y el real. 


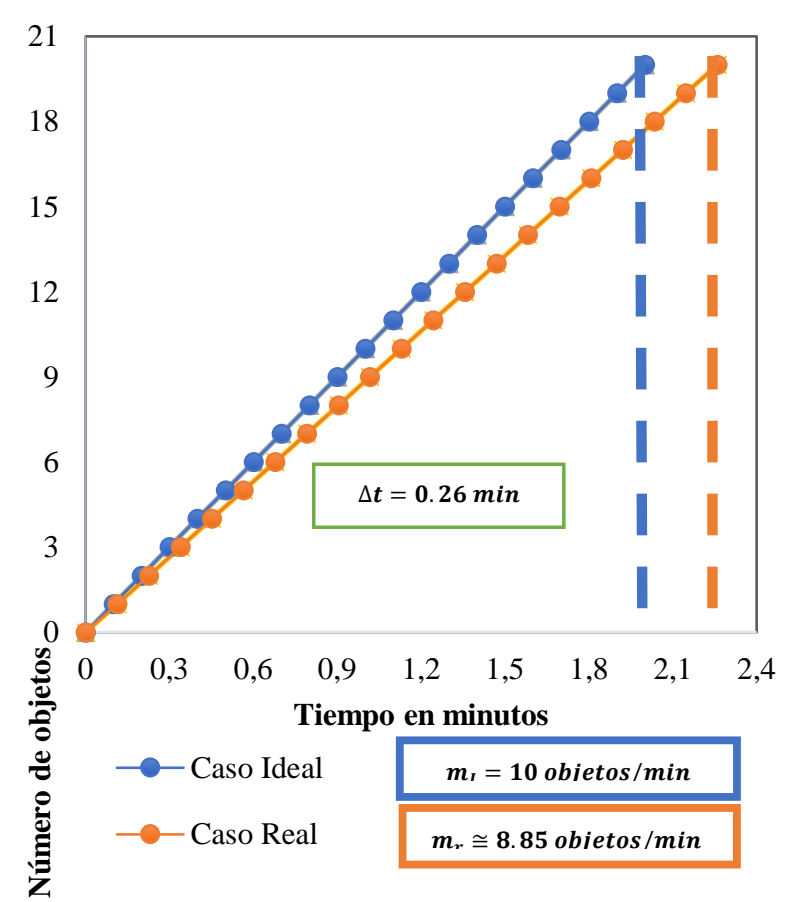

Gráfico 1 Comparación del número de piezas analizadas correctamente con respecto al tiempo

El Gráfico 2 muestra una comparación en porcentaje de la efectividad de análisis para el caso ideal contra el caso real, refiriéndose a la efectividad de objetos analizadas correctamente.

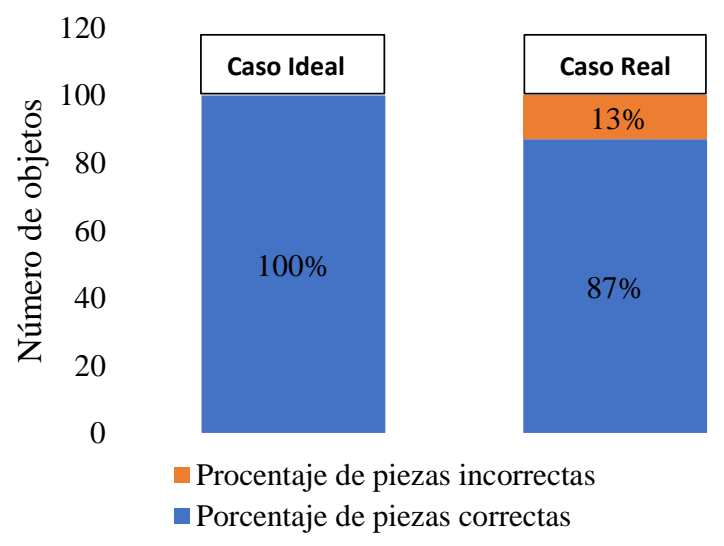

Gráfico 2 Porcentaje de piezas analizadas correctamente en comparación al caso ideal y caso real

\section{Agradecimientos}

Se agradece la contribución de la Universidad Tecnológica de Puebla, por brindar el financiamiento del equipo de National Instruments RoboRIO y los accesorios extras a este. Sin esta contribución no hubiera sido posible este proyecto de investigación.

\section{Conclusiones}

Como se analizó en el capítulo de resultados, se observa que el $87 \%$ de efectividad en el análisis de dimensiones en objetos y colores, es alcanzable con este sistema embebido, siendo la primera etapa de tres. Uno de los puntos débiles encontrados en esta etapa fue, el uso de un sensor ultrasónico HC-SR04, ya que, al ser un sensor con factores de error de $\pm 3 \mathrm{~mm}$ de acuerdo con el fabricante, ocasiono que hubiera un $13 \%$ de mal procesamiento de dimensiones de altura $(\mathrm{Z})$, lo cual, al remplazar este sensor por otro tipo; sensor laser u otro sensor ultrasónico de mejores características, se espera que el procesamiento mejore un $8 \%$ como mínimo, ya que, en el procesamiento de colores y dimensiones en los ejes X y Y, no se presentó ningún porcentaje de error, a pesar de usar una cámara poca resolución.

En función al estado del arte realizado acerca del tema, se resalta que, el desarrollo de sistemas embebidos de visión artificial con hardware RoboRIO aún se encuentra en auge. Por lo que este trabajo, además cubrir el objetivo, pretende enriquecer a los lectores(as), acerca del desarrollo e implementación de sistemas embebidos de visión artificial, basados en LabVIEW y hardware RoboRIO.

\section{Referencias}

National Instruments. (2010), www.ni.com. Obtenido de www.ni.com/fpga: http://www.ni.com/fpga/esa/

National Instruments. (2011). www.ni.com. Obtenido de www.ni.com/white-paper: http://www.ni.com/white-paper/6984/es/\#toc1

National Instruments. (2012). www.ni.com. Obtenido de www.ni.com/white-paper: http://www.ni.com/white-paper/6983/es/

National Instruments. (2017). www.ni.com. Obtenido de forums.ni.com: https://forums.ni.com/t5/FIRST-RoboticsCompetition/FRC-Training-Material-andResources/ta-p/3521059

National Instruments. (2018), www.ni.com. Obtenido de www.ni.com/tutorial: http://www.ni.com/tutorial/14532/en/\#toc15 
Salas Arriarán, S. (2015). Todo Sobre Sistemas Embebidos. Perú: Universidad Peruana de Ciencias Aplicadas.

Silva J., A., Salazar P., M., Ponce M., J., \& Herrera S., G. (2016). Instrumento virtual para diagnosticar y corregir desbalance de maquinaria rotativa. Revista de Tecnología e Innovación Ecorfan-Bolivia, Vol.3 No.7, 73-81.

Silva J., A., Salazar P., M. d., Ponce M., J. J., \& Herrera S., G. (2017). Procedimiento para inspección de tableros eléctricos con termografía infrarroja. Revista de Tecnología e Innovación Vol.4 No.11, 24-35. 